\title{
A novel aggregation approach to reduce complexity of system
}

\section{Sadaf Naqvi*}

Electrical and Electronics Engineering Department, Inderprastha Engineering College,

Ghaziabad, India

Email: sadafnaqvi@rediffmail.com

*Corresponding author

\section{Ibraheem Nasiruddin}

Department of Electrical Engineering,

Jamia Millia Islamia,

New Delhi, India

Email: ibraheem_2k@yahoo.com

\section{Sana Ali}

Department of Electronics and Communication Engineering,

Indraprastha Institute of Information Technology,

Delhi, India

Email: sana@iiitd.ac.in

\begin{abstract}
This paper presents a novel approach to reduce the complexity of the system. In every field of engineering, the analysis and synthesis of the system is an important step. While analysing the system, mathematical modelling is the first step. In actual practice the system model is too complicated to analyse, therefore, model order reduction techniques are applied to analyse such a system. In the present work, an exhaustive study is carried out to design reduced order models for a complex system. Conventional techniques are discussed and applied to two area interconnected power system. A method is proposed and applied to multi-area power system. Dynamic responses of original and reduced models have been compared. Results show that the reduced order model is a good representation of the original higher order model.
\end{abstract}

Keywords: automatic generation control; frequency domain analysis; model order reduction; multivariable system; time domain analysis.

Reference to this paper should be made as follows: Naqvi, S., Nasiruddin, I. and Ali, S. (2020) 'A novel aggregation approach to reduce complexity of system', Int. J. Digital Signals and Smart Systems, Vol. 4, Nos. 1/2/3, pp.100-112.

Biographical notes: Sadaf Naqvi received her Bachelor's in Electrical Engineering from the AMU Aligarh, in 2001, and MTech in Power System, in 2005. She completed her PhD from the Jamia Millia Islamia, New Delhi, in 2014. She is presently working as a Professor at the Electrical and Electronics 
Engineering Department, Inderprastha Engineering College, Ghaziabad. Her area of interest is model order reduction techniques, automatic generation control. She is a life member of IETE and member of IEEE Control System Society.

Ibraheem Nasiruddin received his BSc in Engineering (Hons.), MSc Engineering and $\mathrm{PhD}$ in Electrical Engineering from Aligarh Muslim University, Aligarh, India in 1982, 1987 and 2000, respectively. After serving Delhi Development Authority at Delhi, India for about three years, he had joined the Department of Electrical Engineering, Jamia Millia Islamia in January 1988. Currently, he is working as the Dean, Faculty of Engineering and technology. His current activities include teaching and research in the areas of power system control, optimal control theory, optimal and suboptimal control of power systems, applications of model order reduction techniques and soft computing techniques in power systems and HVDC transmission systems.

Sana Ali graduated in Electrical and Electronics Engineering from the Northern India Engineering College, Guru Gobind Singh Indraprastha University, New Delhi, in 2014. She is presently working as a Jr. Research Engineer from the Department of Electronics and Communication Engineering, Indraprastha Institute of Information Technology, Delhi, New Delhi. She is also pursuing her MTech in Electronics and Communication Engineering General from the same institute.

This paper is a revised and expanded version of a paper entitled 'AGC strategies of power system using aggregation techniques', presented at 2018 IEEE International Conference on Sustainable Energy, Electronics, and Computing Systems (SEEMS-2018), ITS Engineering College, Greater Noida, India, 26-27 October 2018.

\section{Introduction}

In the field of control system a large number of model order reduction techniques are available as mentioned by Singh and Petkar (2010), Vishwakarma (2011), and Komarasamy et al. (2012). However, there is no universal technique which can be applied to every kind of systems. Every technique has its own advantages and disadvantages, over the others. The effectiveness of any reduction technique depends on the way it is utilised and representing the desired characteristics of the system. One of the most popular methods used for model order reduction of large-scale systems has been the aggregation technique introduced in the control system engineering for linear systems by Aoki (1968). Validity of aggregated model is judged by comparing the output response of approximant model with that of the original large model. Further improvement in aggregated model was presented by Joo et al. (2004).

The idea behind the aggregation is very simple and can be understand by considering two sets of variables represented by Sys1 and Sys2. Both the sets of variables represent the physical model of same system but Sys 1 is a set of $n$ variables while Sys 2 is a set of $r$ variables where $r<n$. Then, the set of variables Sys 2 is known as aggregated model of Sys1.

The link between the original model and reduced model is established by a linear transformation. To achieve this aggregation matrix of rank $r$ is obtained. 
Aggregation matrix can be determined by number of ways, some of common methods are:

- continued fraction expansion

- $\quad$ Routh array method

- modal method

- $\quad$ aggregation by using controllability matrix.

\subsection{Continued fraction expansion technique}

Perhaps aggregation by continued fraction expansion has been introduced by Chen and Shieh (1968). This method uses the first and second form of continued fraction expansion. Later, various modified forms of the method were proposed by many authors and applied to MIMO systems also as presented by Prasad et al. (1990), Lucas (1985), Biyık and Arcak (2006), Ishizaki et al. (2014). In 1978, Lamba and Rao suggested a method to obtain aggregation matrix for reduced order model using continued fraction expansion method. Hicken and Sinha (1978) suggested the canonical form for aggregated models.

This technique is explained in detail by Ibraheem et al. (2012). The method is applied to SISO system. Reduced order model is formed using transformation matrix which corresponds to its continued fraction expansion. With the help of this transformation matrix the matrices of reduced order model are obtained. This method is very convenient for computational purpose and also very effective.

\subsection{Aggregation matrix by Routh array}

Another approach to find aggregated model is by using Routh array. The Routh approximant method has an attractive feature that the stability is always guaranteed for the simplified reduced model. It can be utilised to obtain time domain representation of the system analogous to the Routh approximants reported by Naqvi et al. (2014). This method is compared with Pade approximation and applied to a power system model by Singh et al. (2008).

\subsection{Determination of aggregation matrix using eigenvectors/modal matrix}

The aggregation of original model can also be achieved through a modal matrix. This method is called as modal method. In this method approximate model is designed by retaining initial $r$ dominant eigenvalues. Bonvin and Mellichamp (1982) have presented a critical review of model approaches to model reduction. Further improvement in modal analysis is presented by Pottakulath et al. (2013a, 2013b).

The aggregation matrix $K$ consists a set of eigenvectors of original model which is used to determine aggregated model.

\subsection{Aggregation by controllability matrix method}

Aggregation by using controllability matrix has been suggested by Aoki (1968). The aggregation matrix to find the reduced model is calculated with the help of controllability 
matrix of original model. The controllability matrix of original higher order system is determined and then controllability matrix of required reduced order model is assumed by taking arbitrary values of reduced order system matrix. The matrices of reduced order model are chosen in such a way so that they should satisfy the condition of aggregation.

For a controllable system should be of full rank $n$. Hence, by properly choosing $A_{\text {red }}=\operatorname{diag}\left(\lambda_{1}, \lambda_{2}, \ldots \ldots \ldots \lambda_{r}\right)$ and $B_{\text {red }}$ we can make reduced system completely controllable, i.e., the rank of $W_{A_{r}}$ should be $r$.

\section{Proposed method}

The conventional method of aggregation described above when applied on AGC model, it has been observed that most of the roots are complex in nature and they have undesirable effects on system dynamic performance. A new method is proposed to overcome this problem.

Consider a linear controllable large-scale system:

$$
\begin{aligned}
& \frac{d x(t)}{d t}=A x(t)+B u(t), x(0)=x_{0} \\
& y(t)=C x(t)
\end{aligned}
$$

where matrices $A, B, C$ have their usual meaning.

It is desired to describe the time behaviour of:

$$
z(t)=K x(t), z(0)=z_{0}=K\left(x_{0}\right)
$$

where $K$ is a $r \times n$ constant aggregation matrix and vector $z$ is called the aggregation of $x$ and is of $r \times 1$ order. It is desired to maintain the relation (3).

The reduced model matrices can be represented by $A_{\text {red }}, B_{\text {red }}$ and $C_{\text {red }}$. It is assumed that rank $K$ of is $r$.

The pair $\left(A_{\text {red }}, B_{\text {red }}\right)$ should satisfy the perfect aggregation conditions:

$$
\begin{aligned}
& A_{\text {red }} K=K A \\
& B_{\text {red }}=K B \\
& C_{\text {red }} K \cong C
\end{aligned}
$$

Let the controllability matrix of original system is given by:

$$
W_{A} \triangleq\left[\begin{array}{lllll}
B & A B & A^{2} B & \cdots \cdots \cdots & A^{n-1} B
\end{array}\right]
$$

and a modified controllability matrix of reduced order $W_{A_{r}}$.

These matrices are related by:

$$
W_{A_{r}}=K W_{A}
$$

Thus by using pseudo-inverse the aggregation matrix $K$ can be obtained by:

$$
K=W_{A_{r}} W_{A}^{+}
$$


In a proposed method, the aggregation matrix $K$ can be determined from the controllability matrix of original system directly as:

$$
K=W_{A}^{+}(1: r, 1: n),
$$

i.e., by taking elements of $W_{A}^{+}$up to $r^{\text {th }}$ row and $n^{\text {th }}$ column.

In order to determine the matrices of the reduced model the system matrix is obtained from Penrose solvability condition, i.e.,

$$
\begin{aligned}
& A_{\text {red }}=K A K^{T}\left(K K^{T}\right)^{-1} \\
& B_{\text {red }}=K B
\end{aligned}
$$

while matrix $C_{\text {red }}$ can be determined as:

$$
C_{\text {red }}=C K^{T}\left(K K^{T}\right)^{-1}
$$

\section{Model under consideration}

Problem associated with the complexity of the interconnected system is raised in Hasan et al. (2013). The power is generated by various means like, thermal, hydro, nuclear and wind power. But thermal power plants have major share in overall electrical power generation. The modern power plants are running with reheat thermal turbines due to higher efficiency margins as compared to that of non-reheat thermal turbines. For these reasons in the present study an interconnected power system model is considered which consist of reheat thermal turbines. The power plants of model under consideration are interconnected via AC links. The speed governing system and generator in each area are assumed to be associated with single time constants. As reported in $\mathrm{PhD}$ thesis of Ibraheem (1998), the reheat turbine dynamics is represented by a transfer function associated with a double first order time constants due to re-heater dynamics. The models of AC transmission link are also considered to be represented by transfer function with single order time constant. The resulting state space model is of the order of 11 . In present work the model under consideration is simplified by applying aggregation technique. The details of the system are in the next section.

\section{Development of system dynamic model state vectors}

The state vector ' $X$ ' $(11 \times 1)$, control vector ' $U$ ' $(2 \times 1)$ and the disturbance vector ' $W$ ' $(2 \times 1)$ are:

$$
\begin{aligned}
& {[X]=\left[\begin{array}{lllllllllll}
x_{1} & x_{2} & x_{3} & x_{4} & x_{5} & x_{6} & x_{7} & x_{8} & x_{9} & x_{10} & x_{11}
\end{array}\right]^{T} ;} \\
& {[U]=\left[\begin{array}{l}
u_{1} \\
u_{2}
\end{array}\right] ; \quad[W]=\left[\begin{array}{l}
w_{1} \\
w_{2}
\end{array}\right]}
\end{aligned}
$$

System states are: 


$$
\begin{aligned}
& x_{1}=\Delta F_{t 1} ; x_{2}=\Delta P g_{t 1} ; x_{3}=\Delta P R_{t 1} ; \\
& x_{4}=\Delta X g_{t 1} ; x_{5}=\Delta F_{t 2} ; x_{6}=\Delta P g_{t 2} ; \\
& x_{7}=\Delta P R_{t 2} ; x_{8}=\Delta X g_{t 2} ; x_{9}=\Delta P_{t} ; \\
& x_{10}=\int A C E_{t 1} ; x_{11}=\int A C E_{t 2}
\end{aligned}
$$

Control states are:

$$
u_{1}=\Delta P_{C t 1} u_{2}=\Delta P_{C t 2}
$$

Disturbance states are:

$$
w_{1}=\Delta P_{d 1} w_{2}=\Delta P_{d 2}
$$

The structures of state control and disturbance matrices can be derived from the following differential equations obtained from transfer function model.

$$
\frac{d}{d t} \Delta F_{t 1}=\left(\Delta P g_{t 1}-\Delta P_{t}-\Delta P d_{t 1}-\Delta P_{d c}\right)\left(1 / M_{t 1}\right)-\left(D_{t 1} / M_{t 1}\right) \Delta F_{t 1}
$$

where

$$
\begin{aligned}
& M_{t 1}=T p_{t 1} / K p_{t 1}=\frac{2 H_{t 1}}{f^{0}}, D_{t 1}=1 / K p_{t 1} \\
& \frac{d}{d t} \Delta P R_{t 1}=\Delta X g_{t 1}\left(K_{t 1} / T_{t 1}\right)-\Delta P R_{t 1} / T_{t 1} \\
& \frac{d}{d t} \Delta P g_{t 1}=-\Delta P g_{t 1} / T_{r 1}+\Delta P R_{t 1}\left(1 / T_{r 1}-K_{r 1} / T_{t 1}\right)-\Delta X g_{t 1}\left(K_{r 1} K_{t 1} / T_{t 1}\right) \\
& \frac{d}{d t} \Delta X g_{t 1}=\Delta P c_{t 1}\left(K g_{t 1} / T g_{t 1}\right)-\Delta F_{t 1}\left(K g_{t 1} / R_{t 1} T g_{t 1}\right)-\Delta X g_{t 1} / T g_{t 1} \\
& \frac{d}{d t} \Delta F_{t 2}=\left(\Delta P g_{t 2}-a_{12} \Delta P_{t}-\Delta P d_{t 2}-a_{12} \Delta P_{d c}\right)\left(1 / M_{t 2}\right)-\left(D_{t 2} / M_{t 2}\right) \Delta F_{t 2} \\
& \frac{d}{d t} \Delta P R_{t 2}=\Delta X g_{t 2}\left(K_{t 2} / T_{t 2}\right)-\Delta P R_{t 2} / T_{t 2} \\
& \frac{d}{d t} \Delta P g_{t 2}=-\Delta P g_{t 2} / T_{r 2}+\Delta P R_{t 2}\left(1 / T_{r 2}-K_{r 2} / T_{t 2}\right)-\Delta X g_{t 2}\left(K_{r 2} K_{t 2} / T_{t 2}\right) \\
& \frac{d}{d t} \Delta X g_{t 2}=\Delta P c_{t 2}\left(K g_{t 2} / T g_{t 2}\right)-\Delta F_{t 2}\left(K g_{t 2} / R_{t 2} T g_{t 2}\right)-\Delta X g_{t 2} / T g_{t 2}
\end{aligned}
$$




\section{Transfer function model of incremental power flow through EHVAC tie-line $\left(\Delta \boldsymbol{P}_{T}\right)$}

Considering the linearised model of incremental tie-line power flow, the expression for total incremental tie-line power exported from area $i$ can derived as:

$$
\Delta P_{t i}=\sum_{p=1}^{s} 2 \pi T_{i p}\left(\int \Delta F_{t i} d t-\int \Delta F_{t p} d t\right)
$$

where $p$ represents the number of control areas.

For a two-area case one can write:

$$
\Delta P_{t}=2 \pi T_{12}\left(\int \Delta F_{1} d t-\int \Delta F_{t 2} d t\right)
$$

or

$$
\frac{d}{d t} \Delta P_{t}=2 \pi T_{12}\left(\Delta F_{t 1}-\Delta F_{t 2}\right)
$$

For the two identical area interconnected power system,

$$
\begin{aligned}
& \Delta P_{t 1}=\Delta P_{t} \\
& \Delta P_{t 2}=-\left(P_{r 1} / P_{r 2}\right) \Delta P_{t}=a_{12} \Delta P_{t}
\end{aligned}
$$

To incorporate the integral action in the design of optimal AGC regulators, the integrals of area control errors of both areas are derived as follows:

$$
\begin{aligned}
& I A C E_{t 1}=\int\left(\Delta P_{t 1}+B_{t 1} \Delta F_{t 1}\right) d t \\
& \frac{d}{d t}\left(I A C E_{t 1}\right)=\Delta P_{t 1}+B_{t 1} \Delta F_{t 1}
\end{aligned}
$$

Similarly,

$$
\begin{aligned}
& I A C E_{t 2}=\int\left(\Delta P_{t 2}+B_{t 2} \Delta F_{t 2}\right) d t \\
& \frac{d}{d t}\left(I A C E_{t 2}\right)=\Delta P_{t 2}+B_{t 2} \Delta F_{t 2}
\end{aligned}
$$

or

$$
\frac{d}{d t}\left(I A C E_{t 2}\right)=a_{12} \Delta P_{t 2}+B_{t 2} \Delta F_{t 2}
$$

where $a_{12}$ is defined as area size ratio. For identical power system control areas, it is taken as -1 . 
With the help of differential equations (14)-(26) the system can be represented as:

$$
\frac{d x}{d t}=A x+B u+\Gamma w
$$

The matrices $A, B$ and $\Gamma$ can be defined as:

$$
\begin{aligned}
& A=\left[\begin{array}{ccccccccccc}
-\frac{D_{t 1}}{M_{t 1}} & \frac{1}{M_{t 1}} & 0 & 0 & 0 & 0 & 0 & 0 & -\frac{1}{M_{t 1}} & 0 & 0 \\
0 & -\frac{1}{T_{r 1}} & \frac{1}{T_{r 1}}-\frac{K_{r 1}}{T_{t 1}} & \frac{K_{t 1} K_{r 1}}{T_{t 1}} & 0 & 0 & 0 & 0 & 0 & 0 & 0 \\
0 & 0 & -\frac{1}{T_{t 1}} & \frac{K_{t 1}}{T_{t 1}} & 0 & 0 & 0 & 0 & 0 & 0 & 0 \\
-\frac{K g_{t 1}}{R_{t 1} T_{g t 1}} & 0 & 0 & -\frac{1}{T g_{t 1}} & 0 & 0 & 0 & 0 & 0 & 0 & 0 \\
0 & 0 & 0 & 0 & -\frac{D_{t 1}}{M_{t 2}} & \frac{1}{M_{t 2}} & 0 & 0 & -\frac{a_{12}}{M_{t 2}} & 0 & 0 \\
0 & 0 & 0 & 0 & 0 & -\frac{1}{T_{r 2}} & \frac{1}{T_{r 2}}-\frac{K_{r 2}}{T_{t 2}} & \frac{K_{r 2} K_{t 2}}{T_{t 2}} & 0 & 0 & 0 \\
0 & 0 & 0 & 0 & 0 & 0 & -\frac{1}{T_{t 2}} & \frac{K_{t 2}}{T_{t 2}} & 0 & 0 & 0 \\
0 & 0 & 0 & 0 & \frac{K g_{t 2}}{R_{t 2} T g_{t 2}} & 0 & 0 & -\frac{1}{T g_{t 2}} & 0 & 0 & 0 \\
2 \pi T_{12} & 0 & 0 & 0 & -2 \pi T_{12} & 0 & 0 & 0 & 0 & 0 & 0 \\
B_{t 1} & 0 & 0 & 0 & 0 & 0 & 0 & 0 & 1 & 0 & 0 \\
0 & 0 & 0 & 0 & B_{t 2} & 0 & 0 & 0 & a_{12} & 0 & 0
\end{array}\right] \\
& B=\left[\begin{array}{cc}
0 & 0 \\
0 & 0 \\
0 & 0 \\
\frac{K g_{t 1}}{T g_{t 1}} & 0 \\
0 & 0 \\
0 & 0 \\
0 & 0 \\
0 & \frac{K g_{t 2}}{T g_{t 2}} \\
0 & 0 \\
0 & 0 \\
0 & 0
\end{array}\right], \quad \Gamma=\left[\begin{array}{cc}
-\frac{1}{M_{t 1}} & 0 \\
0 & 0 \\
0 & 0 \\
0 & 0 \\
0 & -\frac{1}{M_{t 2}} \\
0 & 0 \\
0 & 0 \\
0 & 0 \\
0 & 0 \\
0 & 0 \\
0 & 0
\end{array}\right]
\end{aligned}
$$




\section{Numerical data}

For the illustration of reduction method considering two identical areas with the following system parameters:

$$
\begin{aligned}
& T_{t 1}=T_{t 2}=0.3 \mathrm{sec} ; \operatorname{Tg}_{t 1}=T g_{t 2}=0.08 \mathrm{sec} ; T_{r 1}=T_{r 2}=10 \mathrm{sec} \\
& K_{r 1}=K_{r 2}=0.5 ; K_{t 1}=K_{t 2}=1.0, K g_{t 1}=K g_{t 2}=1.0 \\
& D_{t 1}=D_{t 2}=0.00833 \text { p.u.MW/Hz; } R_{t 1}=R_{t 2}=2.4 \mathrm{~Hz} / \text { p.u. MW; } \\
& B_{t 1}=B_{t 2}=0.425 \text { p.u.MW/Hz; } M_{t 1}=M_{t 2}=0.167 \text { (p.u. MW) } ; a_{12}=-1 ; \\
& \Delta P d_{t 1}=\Delta P d_{t 2}=0.01 \text { p.u. MW } ; 2 \pi T_{12}=0.545 \text { p.u. MW }
\end{aligned}
$$

With the help of these system data, the coefficient matrices are obtained:

For the application of aggregation method the two reconstructed states are excluded from the above system. Hence the aggregation technique is applied to $9 \times 9$ order original model.

\section{Calculation for reduced order model}

Step 1 Controllability matrix of the original model is calculated from equation (7) and the pseudo inverse of controllability matrix is obtained.

Step 2 Aggregation matrix obtained from the proposed method.

Step 3 The seventh order reduced model matrices $A_{r 7}, B_{r 7}$ and $C_{r 7}$ are obtained from equations (11), (12) and (13) respectively.

The optimal AGC regulators are defined for both original $(9 \times 9)$ order model and the resultant reduced order model, i.e., $(7 \times 7)^{\text {th }}$ order model.

\section{Discussion of simulation results}

Validation of reduced model is done by comparing the dynamic characteristics of original and reduced order model. Simulation results describing the dynamic responses of $\Delta F_{t 1}$ and $\Delta F_{t 2}$ for step input are shown in Figures 1-4. Figures 1 and 2 show the dynamic response of $\Delta F_{t 1}$ when $1 \%$ disturbance is applied to area 1 and area 2 , respectively. Figures 3 and 4 describe the dynamic behaviour of $\Delta F_{t 2}$ under similar disturbances.

From Figure 1, it is evident that for the reduced order system the steady state performance is not matching with the original one. From Figure 2, it is revealed that when disturbance is applied on area 2, the response peak overshoot is less for reduced order model. By investigating Figure 3 and Figure 4 it can be inferred that the reduced order model offers less overshoot for dynamic response of $\Delta F_{t 2}$ when disturbance is applied to area 1 and peak overshoot is more when disturbance is in area 2. However, settling time is comparable. 
Figure 1 Dynamic response of $\Delta F_{t 1}$ for $1 \%$ disturbance in area 1 (see online version for colours)

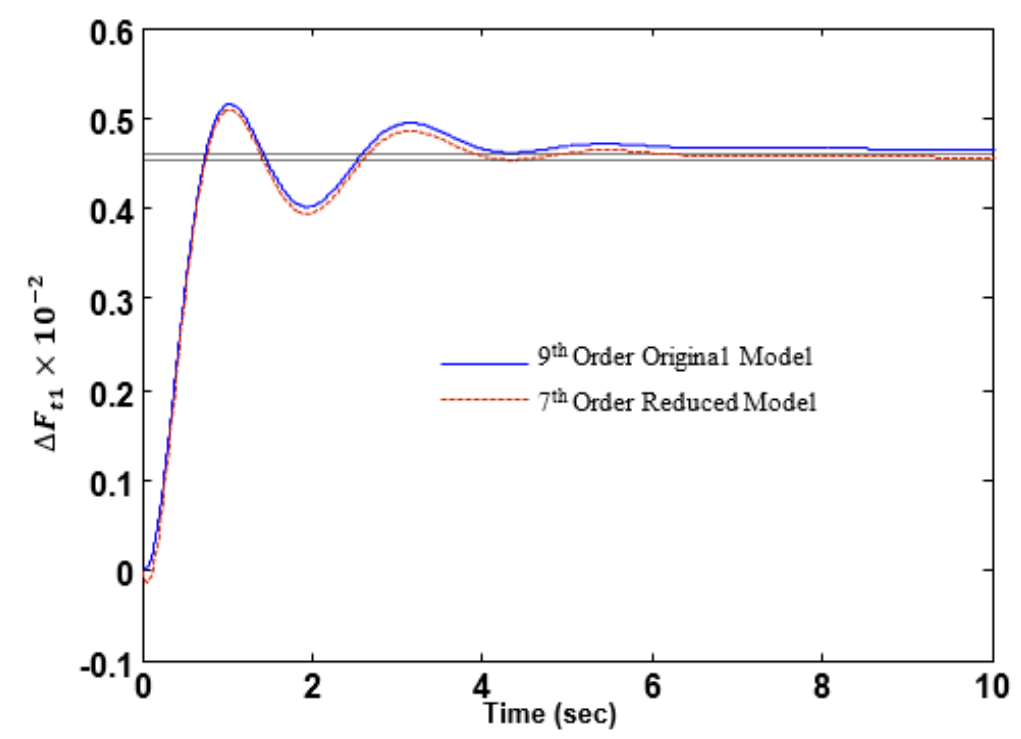

Figure 2 Dynamic response of $\Delta F_{t 1}$ for $1 \%$ disturbance in area 2 (see online version for colours)

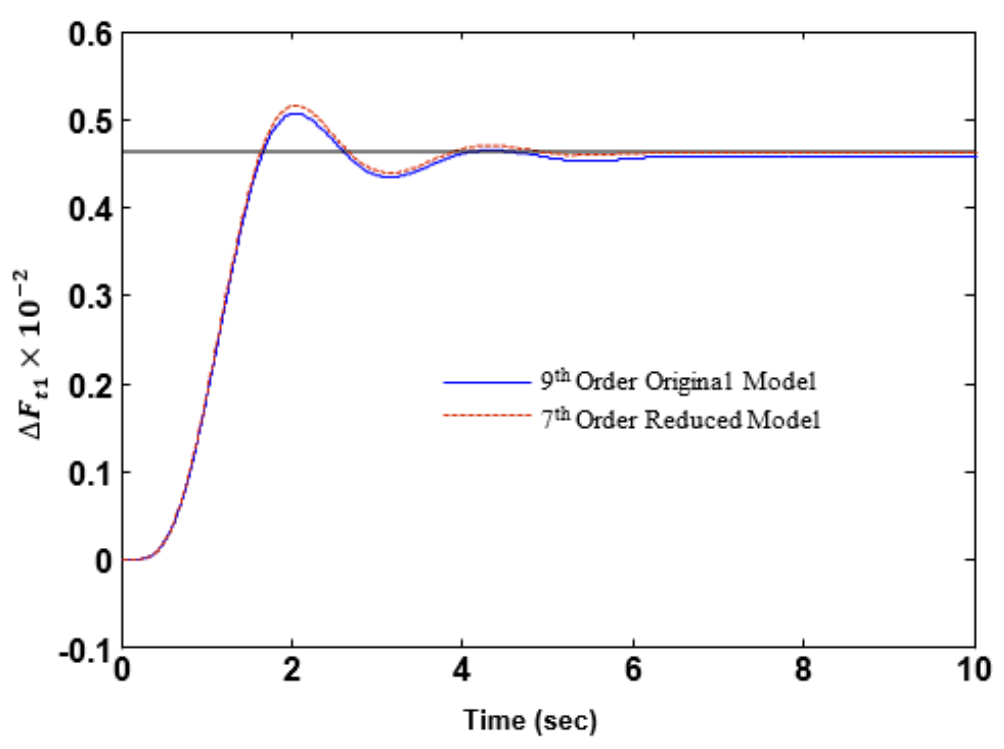


Figure 3 Dynamic response of $\Delta F_{t 2}$ for $1 \%$ disturbance in area 1 (see online version for colours)

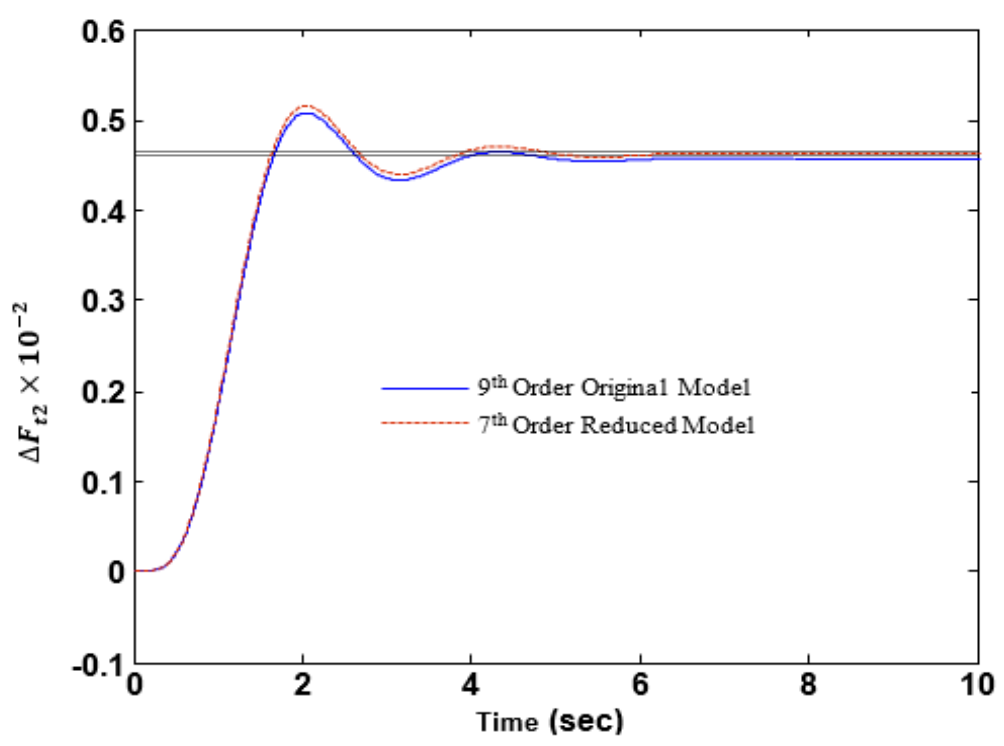

Figure 4 Dynamic response of $\Delta F_{t 2}$ for $1 \%$ disturbance in area 2 (see online version for colours)

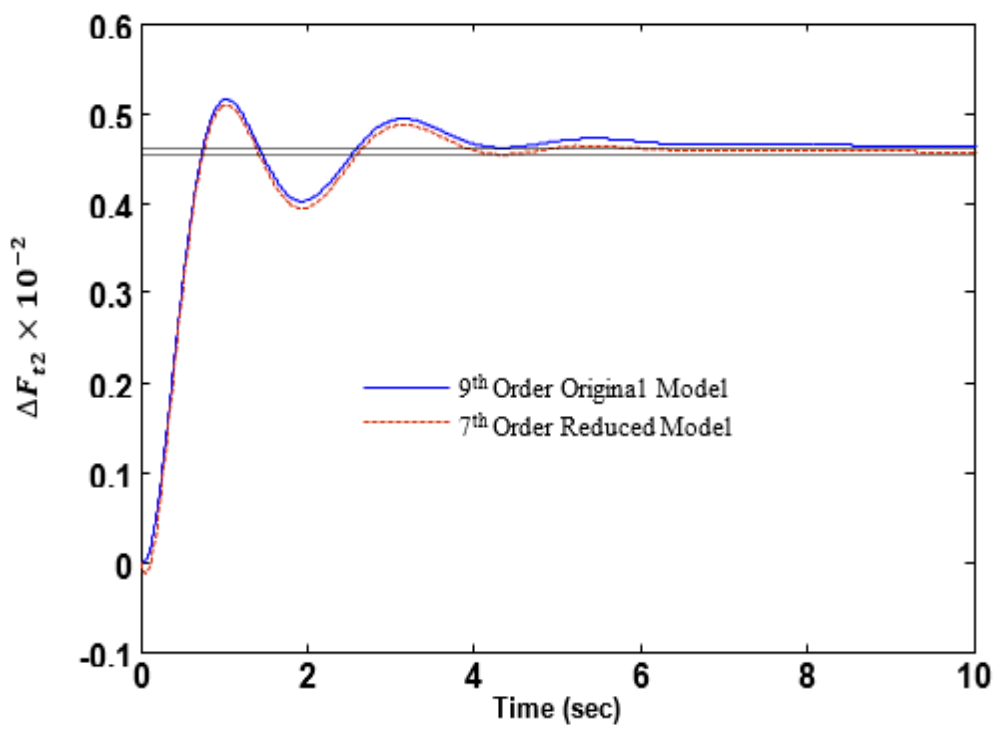




\section{Conclusions}

Through the current study three aggregation methods for model order reduction of linear time-invariant systems have been explained in detail. A new method based on controllability matrix is proposed. The proposed method is in time domain, i.e., the system is represented in state space form. The reduced order models obtained by these reduction methods are retaining main properties of the original high order system and overall show good approximation in the transient as well as steady state periods. The time response of reduced models are compared with original model and found comparable. To see the applicability of these methods, the models of AGC of power system is considered. It is observed in CFE technique that system must be represented in controllable canonical form before application. Conversion of all large-scale system in controllable canonical form is not convenient for most of the system it fails when the system matrix $A$ is a singular matrix or close to singular. In modal aggregation approach, a modal matrix has to be determined which increases the calculations. Application of proposed method on AGC system model shows that the method of aggregation using controllability matrix is better than other methods. As there is no need to find eigenvalues as it was required in modal aggregation method also there is no need to convert system in controllable canonical form unlike the CFE method.

\section{References}

Aoki, M. (1968) 'Control of large dynamic system by aggregation', IEEE Transaction on Automatic Control, Vol. AC-13, No. 3, pp.246-253.

Biylk, E. and Arcak, M. (2006) 'Area aggregation and time scale modeling for sparse nonlinear networks', Proceedings of the 45th IEEE Conference on Decision \& Control, San Diego, CA, USA, 13-15 December.

Bonvin, D. and Mellichamp, D.A. (1982) 'A unified derivation and critical review of modal approaches to model reduction', International Journal of Control, Vol. 35, No. 5, pp.829-848.

Chen, C.F. and Shieh, L.S. (1968) 'A novel approach to linear model simplification', International Journal of Control, Vol. 8, No. 6, pp.561-570.

Hasan, N., Khatoon, S., Ibraheem, Nizamuddin and Singh, Y. (2013) 'Automatic generation control problem in interconnected power systems', Control Theory and Informatics, Vol. 3, No. 2, pp.46-52, ISSN: 2224-5774 (print), ISSNL 2225-0492 (online) [online] http://www.iiste.org.

Hicken, J. and Sinha, N.K. (1978) 'Canonical forms for aggregated models', International Journal of Control, Vol. 27, No. 3, pp.473-485.

Ibraheem (1998) Optimal Control Strategies in Interconnected Power Systems Incorporating AC/HVDC Transmission Links, $\mathrm{PhD}$ thesis, Aligarh Muslim University, Aligarh.

Ibraheem, Kumar, P. and Naqvi, S. (2012) 'Aggregation by continued fraction method of model reduction: an application to SISO system', International Journal of Engineering and Management Research, June, Vol. 2, No. 3, pp.20-23, ISSN no.: 2250-0758.

Ishizaki, T., Kashima, K., Imura, J-i. and Aihara, K. (2014) 'Model reduction and clusterization of large-scale bidirectional networks', IEEE transactions on Automatic Control, January, Vol. 59, No. 1, pp.48-63.

Joo, S-K., Liu, C-C., Jones, L.E. and Choe, J-W. (2004) 'Coherency and aggregation techniques incorporating rotor and voltage dynamics', IEEE Transactions on Power Systems, May, Vol. 19, No. 2, pp.1068-1075. 
Komarasamy, R., Albhonso, N.k. and Gurusamy, G. (2012) 'Order reduction of linear systems with an improved pole clustering', Journal of Vibration and Control, Vol. 18, No. 12, pp.1876-1885.

Lamba, S.S. and Rao, V.S. (1978) 'Aggregation matrix for the reduced order continued fraction expansion model of Chen \& Shieh', IEEE Transaction on Automatic Control, February, Vol. 23, No. 1, pp.81-83.

Lucas, T.N. (1985) 'Model reduction by condensed continued fraction method', Electronics Letters, August, Vol. 21, No. 16, pp.680-681.

Naqvi, S., Ibraheem and Naqvi, S.A. (2014) 'A reduced order model for AGC system using Routh approximation technique', 2014 Innovative Applications of Computational Intelligence on Power, Energy and Controls with their impact on Humanity (CIPECH), Ghaziabad, pp.248-252.

Pottakulath, V., Cheriyan, E.P. and Kumar, R.S. (2013a) 'Determination of reduced order model for tensional interaction investigations using selective modal analysis - part I: initial approach', Electrical Power and Energy Systems, Vol. 44, No. 1, pp.482-487.

Pottakulath, V., Cheriyan, E.P. and Kumar, R.S. (2013b) 'Determination of reduced order model for tensional interaction investigations using selective modal analysis - part II: application to practical power systems', Electrical Power and Energy Systems, Vol. 44, No. 1, pp.454-460.

Prasad, R., Pal, J. and Pant, A.K. (1990) 'Linear multivariable system reduction by continued fraction expansion about a general point 'a', Advances in Modeling and Simulation, Vol. 19, No. 4, pp.47-58, AMSE Press.

Singh, N. and Petkar, S.J. (2010) 'Genatically optimized approximants using dominant pole retention method', International Conference and Workshop on Emerging Trends in Technology (ICWET 2010) - TCET, Mumbai, India.

Singh, N., Prasad, R. and Gupta, H.O. (2008) 'Reduction of power system model using balanced realization, Routh and Pade approximation methods', International Journal of Modelling and Simulation, Vol. 28, No. 1, pp.57-63.

Vishwakarma, C.B. (2011) 'Order reduction using modified pole clustering and Pade approximations', World Academy of Science, Engineering and Technology, Vol. 5, No. 8, pp.998-1002. 